\title{
Giant induced magnetic anisotropy In strain annealed Co-based nanocomposite alloys
}

\author{
Samuel J. Kernion, ${ }^{1, a)}$ Paul. R. Ohodnicki, Jr., ${ }^{1,2}$ Jane Grossmann, ${ }^{1}$ Alex Leary, ${ }^{1}$ \\ Shen Shen, ${ }^{1}$ Vladimir Keylin, ${ }^{3}$ Joseph F. Huth, ${ }^{3}$ John Horwath, ${ }^{4}$ Matthew S. Lucas, ${ }^{4,5}$ \\ and Michael E. McHenry ${ }^{1, b)}$ \\ ${ }^{1}$ Department of Material Science and Engineering, Carnegie Mellon University, 5000 Forbes Ave., Pittsburgh, \\ Pennsylvania 15213, USA \\ ${ }^{2}$ Chemistry and Surface Science Div., National Energy Technology Laboratory (NETL), 626 Cochrans Mill \\ Rd., Pittsburgh, Pennsylvania 15236, USA \\ ${ }^{3}$ Magnetics, Division of Spang and Co., 110 Delta Drive, Pittsburgh, Pennsylvania 15238, USA \\ ${ }^{4}$ Air Force Research Laboratory, Wright-Patterson AFB, Ohio 45433, USA \\ ${ }^{5}$ UTC Inc., 1270 North Fairfield Road, Dayton, Ohio 45432, USA
}

(Received 23 May 2012; accepted 22 August 2012; published online 6 September 2012)

\begin{abstract}
Low loss switching of soft magnetic materials at high frequencies benefits from tuning the induced anisotropy. We show induced anisotropies, $K_{u}$, as large as $1.89 \times 10^{4} \mathrm{~J} / \mathrm{m}^{3}$, developed by strain annealing of Co-rich nanocomposite alloys. Crystalline phases in this alloy system have large negative magnetostrictive coefficients, leading to anisotropy fields per unit stress over twice those developed in FINEMET. Tunable permeability and reduced thicknesses achieved in this process can mitigate eddy-current losses. Giant induced magnetic anisotropies are discussed in light of models for the micromechanisms of amorphous metal deformation, stress-assisted transformations in the crystallites, and directional pair ordering. (C) 2012 American Institute of Physics.

[http://dx.doi.org/10.1063/1.4751253]
\end{abstract}

The magnetic permeability, $\mu$, is the slope of the magnetization curve (Fig. 1) and plays a critical role in determining maxima in: (1) bias operating current, (2) energy stored, and (3) frequency of operation of an inductor. By inducing anisotropy, a linear B-H hysteresis loop can be achieved with constant permeability to a saturating field ( $H_{K}=$ anisotropy field) and magnetization $\left(M_{S}\right)$. A combination of a high $H_{K}$ and constant $\mu$ implies a rotational magnetization process that is less lossy than domain wall motion associated with anomalous eddy-current losses. Two methods of inducing anisotropy are typical: (1) magnetic field processing ${ }^{1,2}$ where an external field is applied in a parallel direction to the core axis and (2) strain annealing in which the sample is placed under tension during crystallization. ${ }^{3}$ We report on large anisotropies achieved by strain annealing of Co-rich nanocomposite alloys. $^{2}$

Induced anisotropy can be exploited to great advantage in tailoring magnetic response. Fig. 1 shows induced transverse anisotropy developed in Co-substituted FINEMET materials annealed under different stress states. The tunability of the permeability by stress annealing is dramatic, where the field over which the permeability is linear increases by nearly an order of magnitude for the sample annealed at $250 \mathrm{MPa}$.

A hierarchy of induced magnetic anisotropies includes:

1. Magnetic pair ordering ${ }^{4}$ where atomic pairs rearrange with bond axes in preferred orientations with respect to the applied field or stress direction (thought to be the operative mechanism in field annealed amorphous alloys). ${ }^{5}$

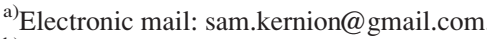

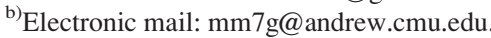

This anisotropy is erasable at relatively low temperatures.

2. Slip-induced anisotropy in crystalline materials ${ }^{6}$ is associated with plastic deformation leading to stress- or strain-induced anisotropy. This results in longer range correlated atomic rearrangement.

The emergence of nanocomposites as premier soft magnetic materials ${ }^{7,8}$ has motivated efforts aimed at elucidating the mechanisms of induced anisotropy. Three archetypes of FeCo-based nanocomposites are near equiatomic FeCobased, Fe-rich, and Co-rich alloys. ${ }^{9}$ Co-rich alloys are of

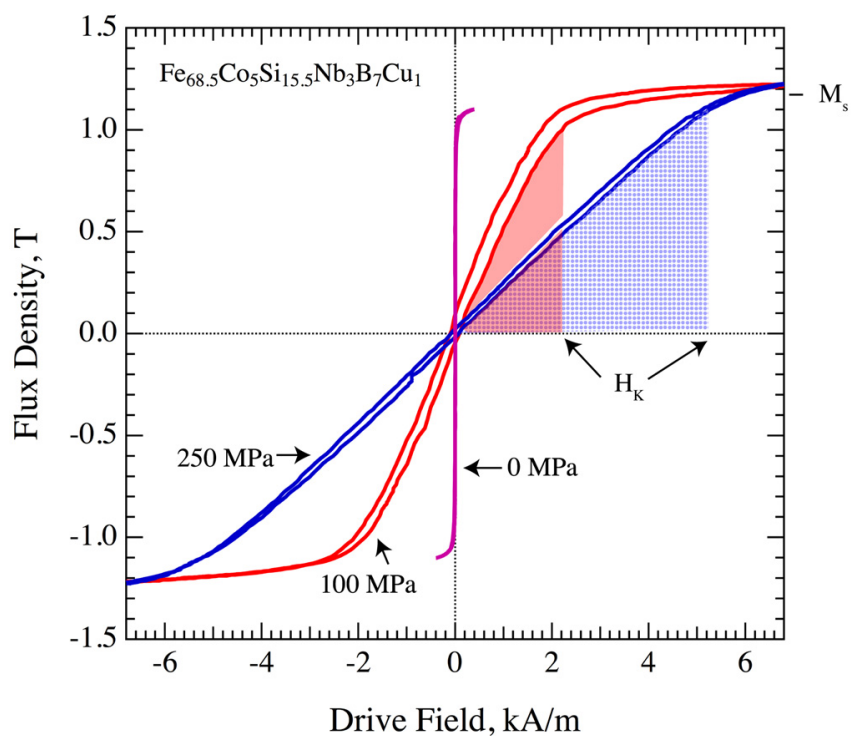

FIG. 1. Hysteresis loops of strain annealed $\mathrm{Fe}_{68.5} \mathrm{Co}_{5} \mathrm{Si}_{15.5} \mathrm{~B}_{7} \mathrm{Nb}_{3} \mathrm{Cu}_{1}$ at a frequency of $1 \mathrm{kHz}$. Shaded regions represent energy stored at the same magnetizations. 
interest because of the competing crystalline phases when the $\mathrm{Co}: \mathrm{Fe}$ ratios are in the two-phase ${ }^{10}$ or single phase regions ${ }^{11}$ on the Co-rich side of the FeCo phase diagram. The kinetics of nanocrystallization events are important to develop a fine microstructure necessary for low losses in nanocomposites. ${ }^{12-16}$

In strain annealed $\mathrm{Fe}_{73.5} \mathrm{Si}_{15.5-x} \mathrm{~B}_{7+x} \mathrm{Nb}_{3} \mathrm{Cu}_{1}$ (FINEMET) ${ }^{17}$ directional pair ordering ${ }^{18}$ and magnetoelastic coupling ${ }^{19}$ are proposed as the origin of the induced anisotropy. The latter theory is well-supported by direct measurement of the elastic strain of crystallites in the direction of the applied stress. ${ }^{20}$ The magnetoelastic theory suggests an unrecoverable plastic strain of the amorphous matrix holds a residual stress on elastically strained crystallites. The induced anisotropy parameter $\left(K_{u}\right)$ in strain annealed FINEMET can be predicted from the residual stress on the crystallites $\left(\sigma_{i}\right)$, the magnetostrictive coeffiecient of the crystallites $\left(\lambda_{s}^{x t a l}\right)$, and the volume fraction crystallized $\left(v_{x t a l}\right)^{19}$

$$
K_{u}=-\frac{3}{2} \lambda_{s}^{x t a l} \sigma_{i} v_{x t a l}
$$

A transverse anisotropy is only developed when $\lambda_{s}^{x t a l}<0$, limiting reduction of Si-content in FINEMET. The only other composition space of Fe-Co binary alloys where $\lambda_{s}^{\text {xtal }}<0$ is the Co-rich side for atomic fractions of $\mathrm{Fe}<0.1$, suggesting that Co-rich alloys may exhibit useful strain annealing response. ${ }^{21}$ The high response to field annealing in Co-rich nanocrystalline alloys also implies a strong possibility that pair ordering could influence the induced anisotropy. Co-based alloys exhibit a higher Curie temperature than Fe-based alloys, increasing the effect that internal fields may have on pair ordering at elevated temperatures. Here, $\left(\mathrm{Co}_{1-x} \mathrm{Fe}_{x}\right)_{89} \mathrm{Zr}_{7} \mathrm{~B}_{4}(\mathrm{x}=0,0.025,0.1)$ with giant induced transverse anisotropy is discussed. The high $K_{u}$ makes Co-based nanocrystalline alloys attractive candidates for high frequency power conversion.

Melt-spinning of $\left(\mathrm{Co}_{1-x} \mathrm{Fe}_{x}\right)_{89} \mathrm{Zr}_{7} \mathrm{~B}_{4}(\mathrm{x}=0,0.025,0.1)$ ribbons was performed in low pressure $\mathrm{Ar}$ on a jet-caster. Alloys from this composition are referred to by their $\mathrm{Co}: \mathrm{Fe}$ ratio. Widths and thicknesses of ribbon varied from 1.9-3 $\mathrm{mm}$ and 30-33 $\mu \mathrm{m}$, respectively. FINEMET-type ribbons of $\mathrm{Fe}_{73.5} \mathrm{Si}_{15.5} \mathrm{~B}_{7} \mathrm{Nb}_{3} \mathrm{Cu}_{1}(\mathrm{FM}-\mathrm{Fe})$ and $\mathrm{Fe}_{68.5} \mathrm{Co}_{5} \mathrm{Si}_{15.5} \mathrm{~B}_{7}$
$\mathrm{Nb}_{3} \mathrm{Cu}_{1}$ (FM-FeCo) were cast on a planar flow caster with a $2.54 \mathrm{~cm}$ width and $20 \mu \mathrm{m}$ thickness. The ribbons were slit to a $3.1 \mathrm{~mm}$ width.

Annealing was performed in a convective tube furnace. A weight was attached to one end of the ribbon and the other end was attached to a winch that allowed the ribbon to be pulled through the furnace at a set speed. Unless otherwise noted, the ribbons traveled at $6 \mathrm{~cm} / \mathrm{min}$, which translates to a period of $240 \mathrm{~s}$ where the ribbon was in the volume of the furnace measured to be at the set temperature. The Fe-rich alloys were annealed at $580^{\circ} \mathrm{C}$ and the Co-rich alloys were annealed at $550^{\circ} \mathrm{C}$ or $560^{\circ} \mathrm{C}$ under various states of tension. The lower temperature for the Co-rich alloys was chosen to limit oxidation. Toroidal cores of the Co-rich compositions were also crystallized at $550^{\circ} \mathrm{C}$ for $3600 \mathrm{~s}$ under zero stress.

Phases were identified by a Panalytical X'pert Pro MPS x-ray diffractometer. JEOL 2000EX and Technai F20 electron microscopes were used to verify phases, estimate grain size, and examine texture and shape anisotropy. A Walker $\mathrm{AMH}$ permeameter measured $\mathrm{BH}$ loops of toroidal cores with an outer diameter of $2.25 \mathrm{~cm}$ at a $1 \mathrm{kHz}$ frequency. The $M_{s}$ of each alloy was measured by a Lake Shore 7407 VSM in a $950 \mathrm{kA} / \mathrm{m}$ applied field.

Hysteresis loops of strain annealed $\mathrm{Co}_{100}, \mathrm{Co}_{97.5} \mathrm{Fe}_{2.5}$, and $\mathrm{Co}_{90} \mathrm{Fe}_{10}$ samples are shown in Fig. 2. All loops show an induced transverse anisotropy relative to furnace annealed counterparts. However, alloys annealed at low stresses exhibit hysteresis loops consistent with transverse striped domain nucleation as saturation approaches, attributed to the large magnitude $\lambda_{\text {xtal }}{ }^{22}$ At low fields in these alloys the magnetization process is likely dominated by lossy domain wall motion. This extends to $\mathrm{Co}_{90} \mathrm{Fe}_{10}$ ribbon annealed at $200 \mathrm{MPa}$ (inset of Fig. 2(c)), although the anisotropy field could still be reasonably measured in this case. In applications, a constant permeability is usually desired. A linear loop was measured for the $300 \mathrm{MPa} \mathrm{Co}_{90} \mathrm{Fe}_{10}$ alloy and the largest thickness reduction of $16 \%$ was observed. In the $\mathrm{C}_{97.5} \mathrm{Fe}_{2.5}$ alloy, when tension is increased to $550 \mathrm{MPa}$ $\left(550^{\circ} \mathrm{C} / 240 \mathrm{~s}\right)$ during annealing, the hysteresis loop exhibits a loss of transverse anisotropy. A second $550 \mathrm{MPa}$ sample $\left(560^{\circ} \mathrm{C} / 480 \mathrm{~s}\right)$, annealed at $560^{\circ} \mathrm{C}$ for twice as long, showed a similar result. Additionally, the $\mathrm{Co}_{97.5} \mathrm{Fe}_{2.5}$ ribbon annealed at $400^{\circ} \mathrm{C}$ under $300 \mathrm{MPa}$ does not have a flat loop,

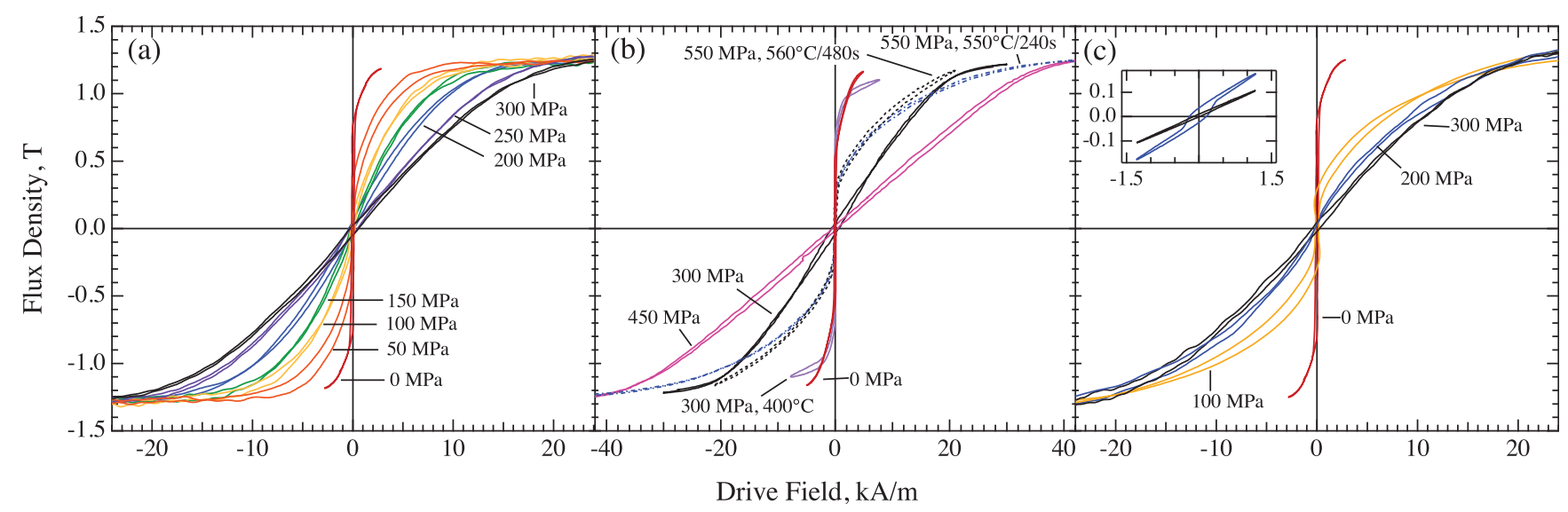

FIG. 2. Hysteresis loops measured at $1 \mathrm{kHz}$, (a) $\mathrm{Co}_{89} \mathrm{Zr}_{7} \mathrm{~B}_{4}$, (b) $\left(\mathrm{Co}_{0.975} \mathrm{Fe}_{0.025}\right)_{89} \mathrm{Zr}_{7} \mathrm{~B}_{4}$, and (c) $\left(\mathrm{Co}_{0.9} \mathrm{Fe}_{0.1}\right)_{89} \mathrm{Zr}_{7} \mathrm{~B}_{4}$. 
suggesting the residual amorphous material is not the direct cause of the induced anisotropy. Coercivity $\left(H_{c}\right)$ for all ribbons, including Fe-based alloys, is shown in Fig. 3(a). A trend with increasing stress is not clearly seen, although the addition of stress to the annealing process increases $H_{c}$ relative to the non-stressed samples of each composition with the exception of $\mathrm{Co}_{100}$.

Saturation magnetizations were unaffected by the stress applied during annealing. Average values of $M_{s}$ for $\mathrm{Co}_{100}$, $\mathrm{Co}_{97.5} \mathrm{Fe}_{2.5}$, and $\mathrm{Co}_{90} \mathrm{Fe}_{10}$ are $1.17 \mathrm{~T}, 1.19 \mathrm{~T}$, and $1.35 \mathrm{~T}$, respectively. The measured $H_{K}, K_{u}$, anisotropy normalized to the applied tension $\left(K_{u} / \sigma\right)$, and $\lambda_{s}^{x t a l}$ as calculated from Eq. (1) (assuming $v_{x t a l}=0.8$ ) are shown in Fig. 3. When the alloys are crystallized under a stress of $300 \mathrm{MPa}$, the $\mathrm{Co}_{97.5} \mathrm{Fe}_{2.5}$ alloy demonstrates the highest $H_{K}$ of $18 \mathrm{kA} / \mathrm{m}$, corresponding to a permeability of $\sim 50$. For cores with linear loops, permeability values ranged from $\sim 150$ for $\mathrm{Co}_{100}$

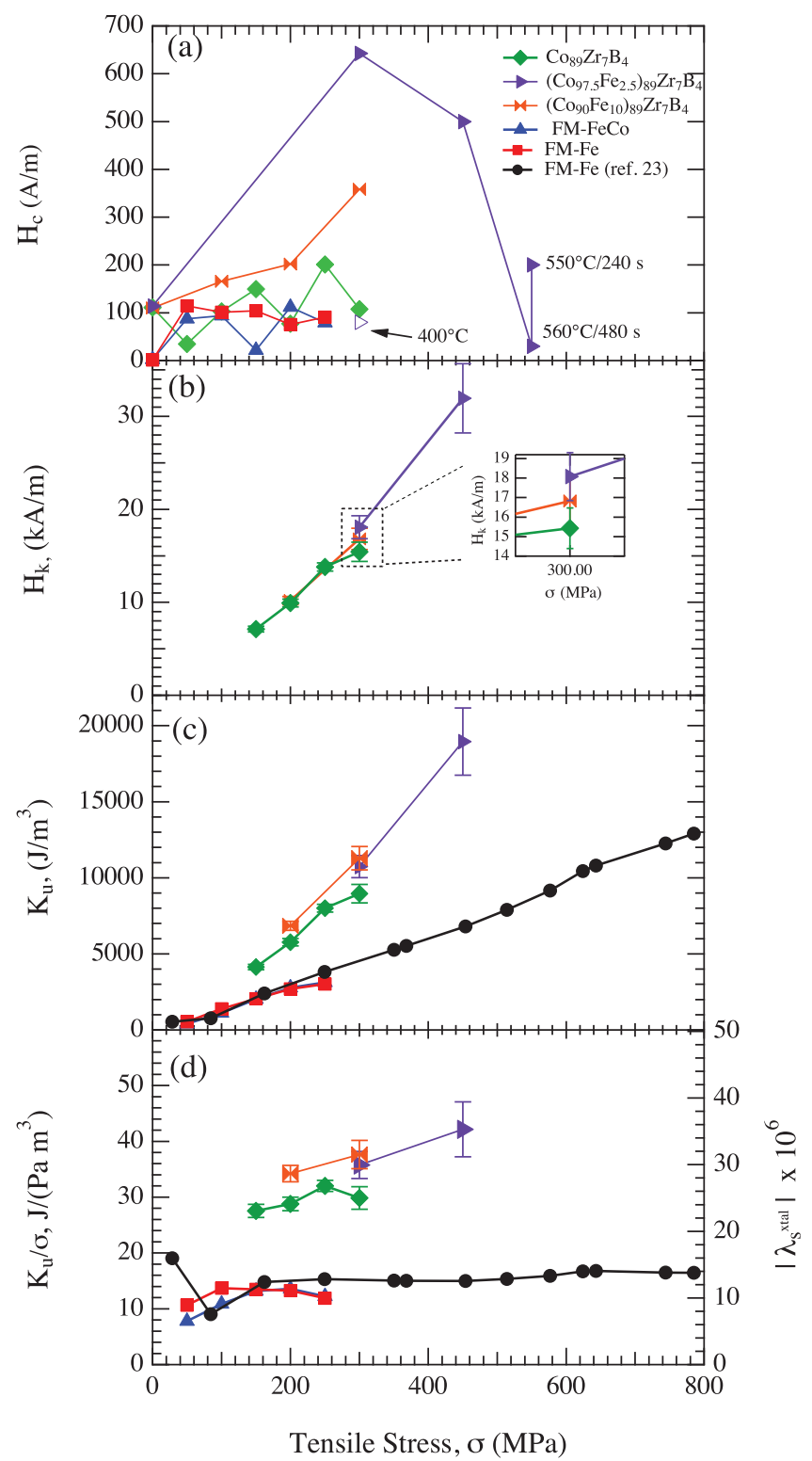

FIG. 3. (a) $H_{c}$ of Fe-based and Co-based alloys, (b) $H_{K}$ of Co-based alloys with inset showing comparison of $300 \mathrm{MPa}$ ribbons, (c) $K_{u}$, and (d) the anisotropy per unit stress $\left(K_{u} / \sigma\right)$ and calculated $\lambda_{s}^{\text {xtal }}$ assuming $v_{x t a l}=0.8$ using Eq. (1). Data on FINEMET included for comparison (from Ref. 23). annealed under $150 \mathrm{MPa}$ of stress to $\sim 30$ for $\mathrm{Co}_{97.5} \mathrm{Fe}_{2.5}$ annealed under $450 \mathrm{MPa}$ of stress. Low permeability values can reduce the need for gapping of cores. Figs. 3(b) and 3(c) include the results on FM-Fe and FM-FeCo measured here and state-of-the-art FINEMET. ${ }^{23}$ The Co-based alloys have a significantly higher $K_{u}$ compared to the Fe-based alloys after similar strain annealing treatments. The $K_{u}$ of 18900 $\mathrm{J} / \mathrm{m}^{3}$ is the highest measured for any strain annealed amorphous or nanocrystalline alloy. The calculated $\lambda_{s}^{x t a l}$ for $\mathrm{Co}_{100}$ agrees well with early ${ }^{21}$ and more recent ${ }^{24}$ measurements for bulk hcp Co. In the latter study, the $\lambda_{s}^{\text {xtal }}$ of the double hexagonal close packed (dhcp) and fcc phases average -10 to $-15 \times 10^{-6}$. A $\lambda_{s}^{x t a l}>0$ is assumed for the bcc phase. Therefore, the high $K_{u}$ of $\mathrm{C}_{97.5} \mathrm{Fe}_{2.5}$ and $\mathrm{Co}_{90} \mathrm{Fe}_{10}$, which are expected to contain phases other than the hcp phase, would require additional sources of anisotropy assuming the bulk $\lambda_{s}^{\text {xtal }}$ applies to the nanocrystallites.

XRD patterns of strain annealed (a) $\mathrm{Co}_{100}$, (b), $\mathrm{Co}_{97.5} \mathrm{Fe}_{2.5}$, and (c) $\mathrm{Co}_{90} \mathrm{Fe}_{10}$ are shown in Figs. 4(a)-4(c). $\mathrm{Co}_{100}$ shows only hcp and fcc phase formation. The crystalline peaks sharpen with applied tension, indicating that grains may be coarsening or the volume fraction crystallized is increasing, which can be attributed to increased atomic mobility provided by the strain ${ }^{25}$ or localized heating. ${ }^{26}$ The $\mathrm{Co}_{97.5} \mathrm{Fe}_{2.5}$ alloys demonstrate hcp, fcc, and bcc phases, consistent with the results of prior work. ${ }^{16,27}$ The $(211)_{b c c}$ peak disappears and the (100) $)_{h c p}$ and (101) $)_{h c p}$ reflections dissipate in the $550 \mathrm{MPa} 550^{\circ} \mathrm{C} / 240 \mathrm{~s}$ sample. A broad peak at $\sim 44.7^{\circ}$ can come from contributions of all three phases. When the intensity of the $450 \mathrm{MPa}$ pattern is subtracted from the $300 \mathrm{MPa}$ pattern, as seen in the inset of Fig. 4(b), the negative peak below $45^{\circ}$ suggests increased fcc or hcp phase formation and the positive peak slightly above $45^{\circ}$ indicates that the bcc phase is diminished in the ribbon annealed under higher stress. Performing the same analysis for the $450 \mathrm{MPa}$ and $550 \mathrm{C} / 240 \mathrm{~s}$ ribbons indicates that the $(101)_{h c p}$ peak has diminished. Coupled with the loss of the $(211)_{b c c}$ peak, the fcc phase is likely the dominant crystalline phase in the 550C/240s sample. The 560C/480s ribbon shows sharpened peaks and the return of the hcp reflections. The $(110)_{b c c}$ peak is reduced to a shoulder on the $(111)_{f c c} /(002)_{h c p}$ peak in this alloy.

A similar trend is seen in the $\mathrm{Co}_{90} \mathrm{Fe}_{10}$ alloy. After $100 \mathrm{MPa}$ strain annealing treatment, only a bcc phase is clearly present. A shoulder on the lower $2 \theta$ side of the peak may indicate the presence of the fcc phase. Increasing the stress to $200 \mathrm{MPa}$ leads to increased shouldering, seen clearly by the subtracted patterns of the 200 and $100 \mathrm{MPa}$ samples (inset of Fig. 4(c)). Increasing the stress to $300 \mathrm{MPa}$ confirms the stabilization of the fcc phase with increasing stress. By Gaussian peak fitting, the integrated intensity of the $(111)_{f c c}$ peak and the $(110)_{b c c}$ peak were measured and the bcc to fcc phase fraction ratio was estimated to be $\sim 1: 1$. Additionally, another shoulder emerges at the $(100)_{h c p}$ peak location, providing the possibility that the hcp phase has increased stability with increasing stress in this composition.

TEM confirmed a nanocrystalline structure with an average grain size of $8.92 \pm 1.2 \mathrm{~nm}$ with no apparent trend with stress. Images and ring diffraction patterns did not provide evidence for crystallographic or shape texturing as sources 


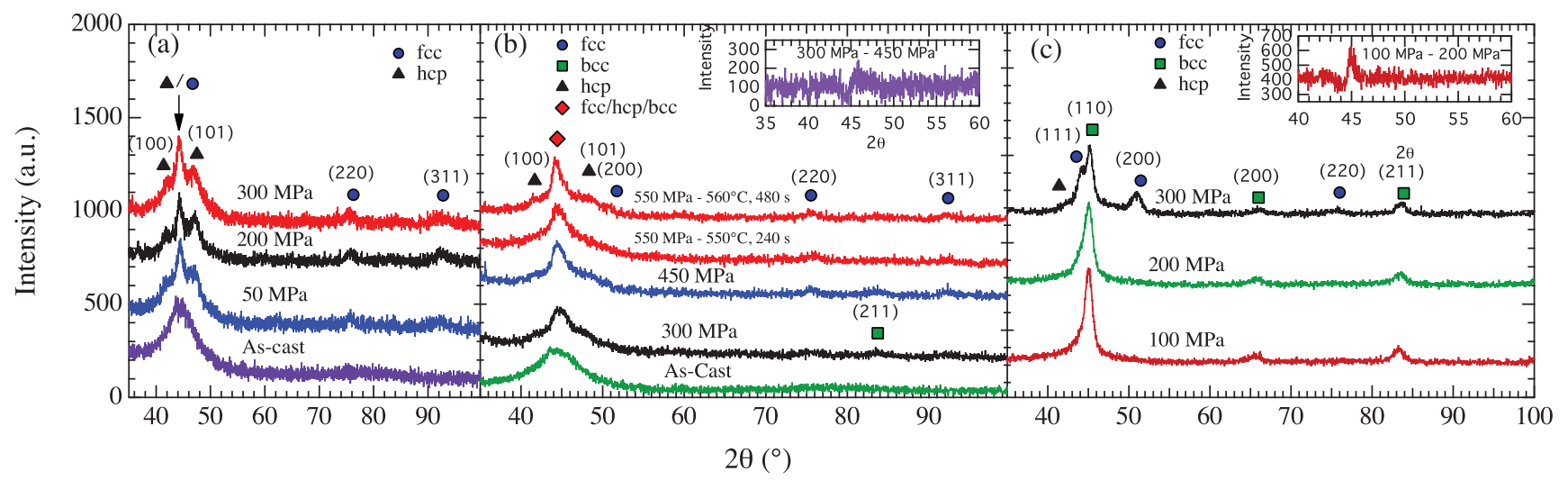

FIG. 4. XRD patterns of strain annealed (a) $\mathrm{Co}_{100}$, (b) $\mathrm{Co}_{97.5} \mathrm{Fe}_{2.5}$, and (c) $\mathrm{Co}_{90} \mathrm{Fe}_{10}$. Unless otherwise noted, the ribbons were annealed at $550{ }^{\circ} \mathrm{C}$. Insets show subtracted patterns.

of the high anisotropy or of being the cause of the decrease in anisotropy in the $550 \mathrm{MPa} \mathrm{Co}_{97.5} \mathrm{Fe}_{2.5}$ ribbon.

The trend of fcc and possible hcp phase stabilization with increased stress can explain the trends in the induced anisotropy. The anisotropy induced in $\mathrm{Co}_{90} \mathrm{Fe}_{10}$ is comparable to $\mathrm{Co}_{97.5} \mathrm{Fe}_{2.5}$ and $\mathrm{Co}_{100}$ when sufficient stress is applied. The increased receptiveness to strain annealing in the $\mathrm{Co}_{90} \mathrm{Fe}_{10}$ alloy coincides with increased fcc phase formation. The bcc phase is still dominant at this stress, however, so the effect of the fcc phase must be large relative to the volume fraction of the phase present. This suggests either very large intrinsic $\lambda_{s}^{x t a l}$ or significant pair ordering unique to the fcc phase as compared to the bcc phase. Further complicating the analysis are the $\mathrm{Co}_{97.5} \mathrm{Fe}_{2.5} 550 \mathrm{MPa}$ samples, which exhibit fcc and hep crystalline phases and a lower $K_{u}$ than the $450 \mathrm{MPa}$ sample. This could be due to secondary phase formation or excessive crystallization leading to an inability to retain elastic strain on the grains, but secondary crystalline phases are not seen in the XRD or TEM results and an amorphous halo is still seen in the XRD patterns.

Another possibility for the loss of anisotropy is a martensitic transformation of (1) bcc $\rightarrow$ fcc or (2) bcc $\rightarrow$ hcp. This is consistent with the results seen in Fe-Ga and Co-Fe at phase boundary compositions. ${ }^{28-30}$ A similar phenomenon is believed to occur in the Co-rich alloys, where a parent phase undergoes a displacive transformation. The positive Bain strain along the $[001]_{b c c}$ direction in the bcc $\rightarrow$ fcc transformation would couple with the positive $\lambda_{001}^{b c c}$, inducing an easy direction along the direction of strain. Assuming this is true, the hysteresis loop measured after $550 \mathrm{MPa}$ strain annealing, where the the bcc phase fraction has been diminished, could be attributed to the bcc $\rightarrow$ fcc martensitic transformation.

A martensitic transformation of fcc $\leftrightarrow$ hcp could also be used to explain the high $K_{u}$ seen in the Fe-containing Co-rich alloys, such as the $300 \mathrm{MPa} \mathrm{Co}_{90} \mathrm{Fe}_{10}$ alloy. Both transformations are well known in Co and Co-based alloys and both transformations are capable of straining the material in the direction of the applied stress. ${ }^{31}$ In both cases, the large strains accompanied with the transformation would couple with the negative $\lambda_{s}^{x t a l}$ of the parent phase to result in an induced transverse anisotropy. Pair ordering between $\mathrm{Fe}$ and Co should also be enhanced with a higher Fe-content in the $\mathrm{Co}_{90} \mathrm{Fe}_{10}$ alloy and may significantly contribute to the induced anisotropy.
To conclude, $\left(\mathrm{Co}_{1-x} \mathrm{Fe}_{x}\right)_{89} \mathrm{Zr}_{7} \mathrm{~B}_{4} \quad(\mathrm{x}=0,0.025,0.1)$ nanocrystalline alloys were reported with huge induced anisotropy through strain annealing, with the largest $K_{u}$ of $18900 \mathrm{~J} / \mathrm{m}^{3}$ found in the $\left(\mathrm{Co}_{0.975} \mathrm{Fe}_{0.025}\right)_{89} \mathrm{Zr}_{7} \mathrm{~B}_{4}$ alloy crystallized under $450 \mathrm{MPa}$ of tension. The results generally support the position that magnetoelastic coupling is the primary source of anisotropy as originally proposed for FINEMET alloys. ${ }^{19}$ Tension applied during crystallization effects phase stability in the Co-rich Fe containing alloys, which directly impacts the anisotropy. With a huge induced anisotropy, Cobased nanocrystalline alloys can be utilized in applications requiring a tunable permeability, i.e., power conversion applications.

S.J.K. and M.E.M. gratefully acknowledge support from the Army Research Laboratory through Cooperative Agreement W911NF-08-2-0024.

${ }^{1}$ F. Johnson, H. Garmestani, S. Chu, M. McHenry, and D. Laughlin, IEEE Trans. Magn.40, 2697 (2004).

${ }^{2}$ P. Ohodnicki, J. Long, D. Laughlin, M. McHenry, V. Keylin, and J. Huth, J. Appl. Phys. 104, 113909 (2008).

${ }^{3}$ M. Ohnuma, K. Hono, T. Yanai, H. Fukunaga, and Y. Yoshizawa, Appl. Phys. Lett. 83, 2859 (2003).

${ }^{4}$ L. Néel, J. Phys. Radium 15, 225 (1954).

${ }^{5} \mathrm{R}$. Hasegawa, in Properties and Applications of Nanocrystalline Alloys from Amorphous Precursors, edited by M. M. B. Idzikowski and P. Svec (Kluwer Academic Pub, Dordrecht, Netherlands, 2005), p. 189.

${ }^{6}$ S. Chikazumi and C. Graham, Physics of Ferromagnetism (Oxford University Press, New York, 2009), Vol. 94.

${ }^{7}$ M. McHenry, M. Willard, and D. Laughlin, Prog. Mater. Sci. 44, 291 (1999).

${ }^{8}$ M. McHenry and D. Laughlin, Acta Mater. 48, 223 (2000).

${ }^{9}$ F. Johnson, C. Um, M. McHenry, and H. Garmestani, J. Mag. Mag. Mater. 297, 93 (2006).

${ }^{10}$ P. Ohodnicki, S. Park, H. McWilliams, K. Ramos, D. Laughlin, and M. McHenry, J. Appl. Phys. 101, 09N108 (2007).

${ }^{11}$ P. Ohodnicki, Jr., Y. Qin, M. McHenry, D. Laughlin, and V. Keylin, J. Mag. Mag. Mater. 322, 315 (2010).

${ }^{12}$ A. Hsiao, S. Turgut, M. A. Willard, E. Selinger, M. Lee, D. E. Laughlin, M. E. McHenry, and R. Hasegawa, MRS Res. Symp. Proc. 577, 551 (1999).

${ }^{13}$ A. Hsiao, M. McHenry, D. Laughlin, M. Kramer, C. Ashe, and T. Ohkubo, IEEE Trans. Magn. 38, 3039 (2002).

${ }^{14}$ M. McHenry, F. Johnson, H. Okumura, T. Ohkubo, V. Ramanan, and D. Laughlin, Scr. Mater. 48, 881 (2003).

${ }^{15}$ P. Ohodnicki, Jr., D. Laughlin, M. McHenry, and M. Widom, Acta Mater. 58, 4804 (2010).

${ }^{16}$ S. Kernion, P. Ohodnicki, and M. McHenry, J. Appl. Phys. 111, 07A316 (2012). 
${ }^{17}$ L. Kraus, K. Závta, O. Heczko, P. Duhaj, G. Vlasak, and J. Schneider, J. Magn. Magn. Mater. 112, 275 (1992).

${ }^{18}$ B. Hoffmann and H. Kronmüller, J. Magn. Magn. Mater. 152, 91 (1996).

${ }^{19} \mathrm{G}$. Herzer, IEEE Trans. Magn. 30, 4800 (1994).

${ }^{20}$ M. Ohnuma, K. Hono, T. Yanai, M. Nakano, H. Fukunaga, and Y. Yoshizawa, Appl. Phys. Lett. 86, 152513 (2005).

${ }^{21}$ S. Williams, Rev. Sci. Instrum. 3, 675 (1932).

${ }^{22}$ A. Hubert and R. Schäfer, Magnetic Domains: The Analysis of Magnetic Microstructures (Springer, Heidelberg, NY, 1998).

${ }^{23}$ G. Herzer, V. Budinsky, and C. Polak, J. Phys.: Conf. Ser. 266, 012010 (2011).

${ }^{24}$ T. Wakiyama, H. Brooks, E. Gyorgy, K. Bachmann, and D. Brasen, J. Appl. Phys. 49, 4158 (1978).
${ }^{25}$ C. Schuh, T. Hufnagel, and U. Ramamurty, Acta Mater. 55, 4067 (2007). ${ }^{26}$ J. Lewandowski and A. Greer, Na. Mater. 5, 15 (2005).

${ }^{27}$ P. Ohodnicki, Jr., V. Keylin, H. McWilliams, D. Laughlin, and M. McHenry, J. Appl. Phys. 103, $07 E 740$ (2008).

${ }^{28}$ A. Khachaturyan and D. Viehland, Metall. Mater. Trans. A 38, 2308 (2007).

${ }^{29}$ A. Khachaturyan and D. Viehland, Metall. Mater. Trans. A 38, 2317 (2007).

${ }^{30}$ D. Hunter, W. Osborn, K. Wang, N. Kazantseva, J. Hattrick-Simpers, R. Suchoski, R. Takahashi, M. Young, A. Mehta, L. Bendersky et al., Nat. Commun. 2, 518 (2011).

${ }^{31}$ Y. Liu, H. Yang, G. Tan, S. Miyazaki, B. Jiang, and Y. Liu, J. Alloys Compd. 368, 157 (2004). 